\title{
Permanent Magnet Synchronous Machine Torque Estimation Using Low Cost Hall-Effect Sensors
}

\author{
Daniel Fernández Alonso \\ University of Oviedo \\ Gijon, Spain \\ dflaborda@uniovi.es
}

\author{
YeGu Kang \\ WEMPEC \\ University of Wisconsin Madison \\ Wisconsin, USA \\ ykang22@wisc.edu \\ David Díaz Reigosa \\ University of Oviedo \\ Gijon, Spain \\ diazdavid@uniovi.es
}

\author{
Diego Fernández Laborda \\ University of Oviedo \\ Gijon, Spain \\ dflaborda@uniovi.es
}

Fernando Briz

University of Oviedo

Gijon, Spain

fernando@isa.uniovi.es

\author{
María Martínez Gómez \\ University of Oviedo \\ Gijon, Spain \\ martinezgmaria@uniovi.es
}

\begin{abstract}
Torque measurement/estimation in permanent magnet synchronous machines (PMSMs) is required in a large variety of applications. Direct torque measurement by means of torque transducers is typically limited to laboratory applications due to cost, reliability and room concerns. Alternatively, torque can be estimated. Torque estimation methods require accurate knowledge of machine parameters. Injection of a high frequency (HF) signal in the stator via inverter has been shown to be a viable option for online machine parameters identification in PMSMs. However, this introduces concerns on the performance of the machine due to the noise, vibration and additional losses produced by the HF signal. This paper proposes a torque estimation method for PMSMs using low cost Hall-effect sensors. The method is based on the dependency of the leakage flux complex vector due to interaction between permanent magnet flux and stator coil flux and does not interfere with the drive operation of the machine.
\end{abstract}

Keywords-Torque estimation, Hall-Effect sensors, leakage flux complex vector, PMSM leakage flux.

\section{INTRODUCTION}

Permanent magnet synchronous machines (PMSMs) are commonly used in industrial automation for traction, robotics, wind energy or aerospace applications. The use of PMSMs has been gradually increasing and replacing other machine types due to their power density, torque capabilities, dynamics and efficiency [1], [2]. Accurate control, diagnostics and monitoring of PMSM drives, are required in industrial applications. Machine torque is a variable required to e.g., enhance controllability [3], find the maximum torque per ampere (MTPA) trajectory [4], early fault detection [5], or as a redundant parameter for improving safety [6]. In electric vehicles (EV) or hybrid-electric vehicles (HEVs), accurate

This work was supported in part by the Research, Technological Development and Innovation Programs of the Spanish Ministry Economy and Competitiveness, under grant MINECO-17-ENE2016-80047-R and by the Government of Asturias under project IDI/2018/000188 and FEDER funds. torque measurement/estimation is required for the implementation of torque vectoring [7], anti-lock braking system (ABS), vehicle stability control (VSC) or anti-slip regulator (ASR) controller [8].

PMSMs torque can be measured or estimated. Torque measurement systems can be roughly classified according to the measured variable: torsional strain between shafts or torsional displacement of a shaft. Torque transducers measure torsional strain between two rotating shafts, typically using strain gauges [9],[10], whilst torsional displacement is measured using optical sensors [11], [12]. Measured signals can be transmitted to the stationary part using slip rings, optical grating or wirelessly. However, all torque measurement systems are typically expensive and bulky. Also, they lower the robustness of the drive and their performance can be compromised by environmental conditions e.g., due to electromagnetic interference. Torque transducers also require well-balanced mechanical coupling systems which extend the shaft length, increasing the system inertia and modifying mechanical resonances. For these reasons, torque transducers are typically limited to laboratory and test equipment. Torque estimation techniques are therefore the preferred option for industrial applications.

A variety of PMSMs torque estimation methods have been proposed, including those based on the general torque equation; use of neural networks; use of flux observers; and use of high frequency signal injection. General torque equation-based methods require accurate knowledge of machine parameters, e.g., permanent magnet flux linkage or the inductances [14], [15], which will vary with machine operating conditions. Torque estimation algorithms based on neural networks require large computational capability and are also parameter dependent [16]. Flux observers have been used for torque estimation purposes [17], [18], their main disadvantage being the machine 
parameters dependency. PMSMs parameters can also be estimated by means of high frequency signal injection-based methods [19], [20], which are independent of machine working conditions. The main drawbacks of these techniques are the additional losses, noise and vibration induced due to the injected HF signal. Table I summarizes advantages and disadvantages of the torque estimation methods discussed above.

Table I. Torque measurement/estimation methods

\begin{tabular}{|c|c|c|c|c|c|}
\hline & Cost & $\begin{array}{c}\text { Parameter } \\
\text { dependency }\end{array}$ & $\begin{array}{c}\text { Computational } \\
\text { time }\end{array}$ & Robustness & Accuracy \\
\hline $\begin{array}{c}\text { Torque } \\
\text { transducers }\end{array}$ & $\begin{array}{c}\text { Very } \\
\text { High }\end{array}$ & Null & Null & Very Low & Very High \\
\hline $\begin{array}{c}\text { Torque } \\
\text { Equation } \\
{[14],[15]}\end{array}$ & Null & Very High & Very Low & Very High & Low \\
\hline $\begin{array}{c}\text { Neural } \\
\text { networks } \\
{[16]}\end{array}$ & Null & Low & Very High & Very High & Medium-High \\
\hline $\begin{array}{c}\text { Observer } \\
{[17],[18]}\end{array}$ & Null & High & High & Very High & Medium \\
\hline $\begin{array}{c}\text { HF injection } \\
{[19][20]}\end{array}$ & Null & High & High & Very High & Medium \\
\hline $\begin{array}{c}\text { Hall-sensor } \\
\text { based }\end{array}$ & Null* & High & Very Low & High & Medium \\
\hline
\end{tabular}

* Cost of the proposed system will be null in case the PMSM is equipped with linear Hall sensors.

This paper proposes a method for torque estimation using the flux measurements provided by linear Hall-effect sensors [21][25]. Hall-effect sensors are typically used in PMSM for initial position estimation including polarity [21]-[25], but can also be used for diagnostic and monitoring purposes e.g., PM temperature estimation [26], rotor load defects detection [27] or improve fault tolerant capability [28]. The proposed method is based on the dependency of the flux measured by the Hall-effect sensors on the PM flux and the stator coils currents. Hall-effect sensors located in the stator provide stationary measurement of PM leakage flux which is affected by $d$ - and $q$-axes currents in a different manner: flux weakening current changes its amplitude while torque current shifts PM flux lines to induce torque [26]. The proposed method is insensitive to magnet temperature or flux-weakening current and can be applied to both surface permanent magnet synchronous machines (SPMSMs) and interior permanent magnet synchronous machines (IPMSMs) [29].

This paper is organized as follows: Hall-effect sensor-based flux leakage measurement in PMSMs is presented in section II, torque estimation using leakage flux is presented in section III, experimental results are provided in section IV, and conclusions are finally presented in section $\mathrm{V}$.

\section{Hall-EfFect Sensor-Based LeaKage FluX MEASUREMENT IN PMSMS}

This section describes the leakage flux measurement system in PMSMs. Fig. 1 shows the IPMSM and the SPMSM designs that will be used for the analysis of the proposed method. The
IPMSM is a prototype and the SPMSM is commercial machine from ABB. Their main characteristics are shown in Table II. a)

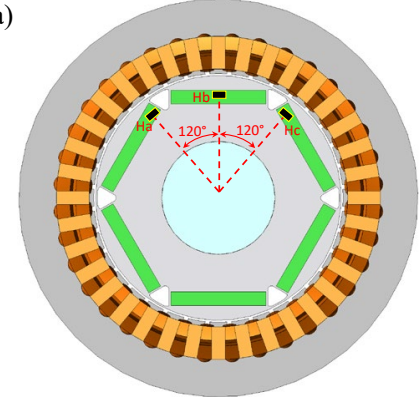

c)

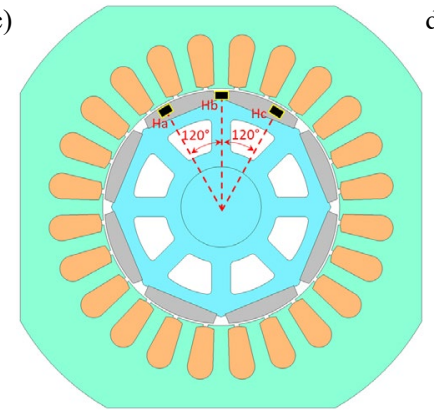

b)

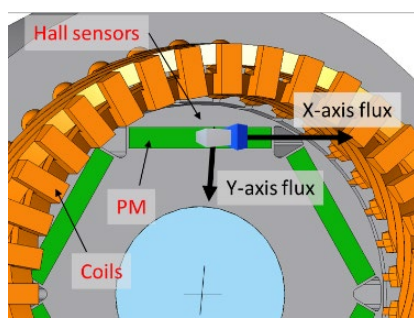

d)

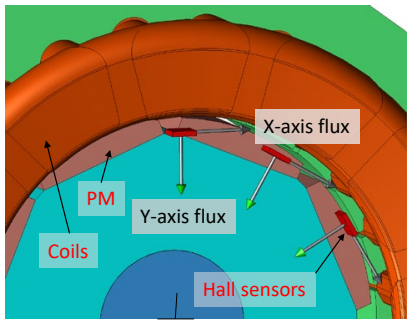

Fig. 1. Schematic representation and sensors location. a) 2D and b) 3D for the IPMSM; c) 2D and d) 3D for the SPMSM; $x$ and $y$ correspond to the tangential and radial directions, respectively.

Fig. 1a) and 1c) show the location of the three Hall-effect sensors $(H a, H b$ and $H c)$ in each machine. Fig. $1 \mathrm{~b})$ and $1 \mathrm{~d})$ show the coordinate system, with $x$-axis being aligned with the rotor tangential direction and $y$-axis being aligned with the radial direction. Axial flux (z-axis) does not contain useful information for torque estimation purposes at the current location and will not be further referenced. The phase shift among sensors is 120 electrical degrees. This corresponds to 40 mechanical degrees in the 6-pole IPMSM in Fig. 1a), and 30 mechanical degrees in the 8-pole SPMSM in Fig. 1c).

Table II. Electric and geometric machine parameters

\begin{tabular}{|l|l|l|}
\hline & SPMSM & IPMSM \\
\hline$P_{\text {rated }}(\mathrm{kW})$ & 6.6 & 7.5 \\
\hline$I_{\text {rated }}(\mathrm{A})$ & 15 & 14 \\
\hline$n_{\text {rated }}(\mathrm{rpm})$ & 1000 & 1000 \\
\hline Stator slots & 24 & 36 \\
\hline Number of poles & 8 & 6 \\
\hline Rotor radius (mm) & 57 & 54.2 \\
\hline Magnets & - & $\mathrm{N} 42 \mathrm{SH}$ \\
\hline $\begin{array}{l}\text { Magnet dimensions: width, height } \\
\text { and length (mm) }\end{array}$ & $36 \times 9 \times 28$ & $42 \times 6 \times 50$ \\
\hline $\begin{array}{l}\text { Magnet position from shaft center } \\
\text { (mm) }\end{array}$ & 52.5 & 44.6 \\
\hline Hall sensor position, $x y z(\mathrm{~mm})$ & $0,0,5$ & $0,0,5$ \\
\hline Airgap length (mm) & 1.2 & 0.4 \\
\hline Inner stator radius (mm) & 59.4 & 55 \\
\hline Outer stator radius $(\mathrm{mm})$ & 93 & 88 \\
\hline Stack length (mm) & 84 & 100 \\
\hline
\end{tabular}

Fig. 2 shows flux lines distribution on $y-z$ plane of the IPMSM obtained by means of finite element analysis (FEA). It can be observed that the maximum concentration of leakage 
flux lines occurs at the edge of the permanent magnet (PM). Therefore, this is considered the most suitable location for the Hall-effect sensors.

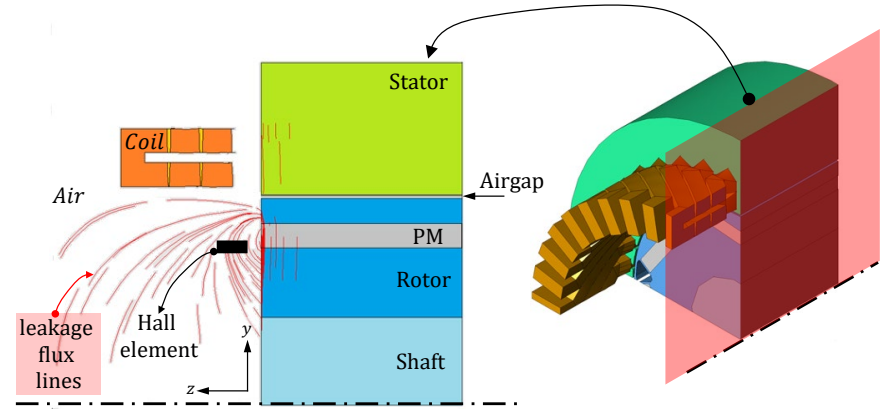

Fig. 2. FEA results. Leakage flux on $y-z$ plane under no load conditions and Hall sensor location.

An example of the FEA calculated magnetic leakage flux density along the $x$-axis direction (tangential) and $y$-axis direction (radial) seen by the sensors during a rotor revolution is shown in Fig. 3. It can be observed that the flux at the sensors' location consist of quasi-sinusoidal fundamental wave plus harmonics, the phase shift of the fundamental wave among sensors being $120^{\circ}$. It is observed that the magnitude in the radial direction is around twice of that in the tangential direction. Therefore, radial flux measurements (i.e. $y$-axis) will be used in this paper for torque estimation purposes.
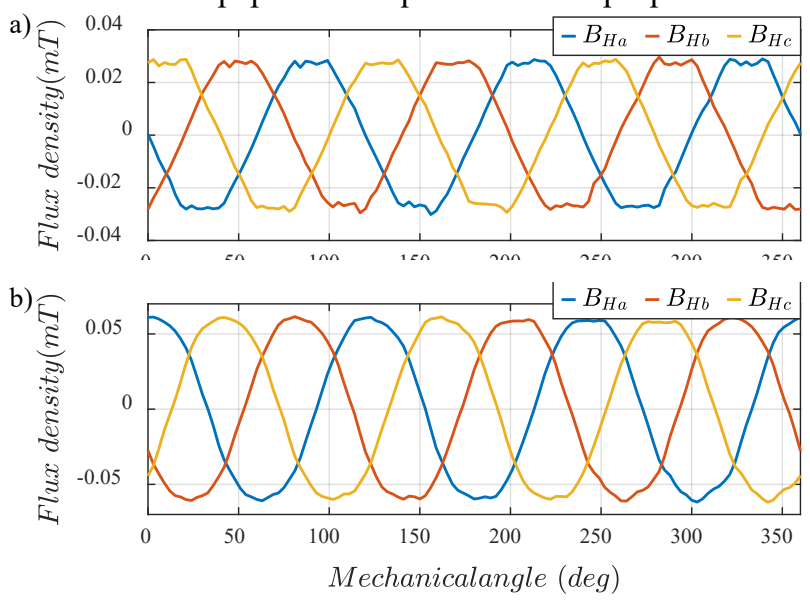

Fig. 3.FEA results. Flux density at sensors location for the IPMSM case: a) tangential ( $x$-axis) and b) radial direction ( $y$-axis).

\section{Torque Estimation Using The Measured Leakage FLUX BY HALL-EFFECT SENSORS}

\section{a. Torque estimation}

The total torque induced in a PMSM is defined by (1), where $p$ is the number of pole pairs of the machine, $\lambda_{d s}^{r}$ and $\lambda_{q s}^{r}$ are the $d$ - and $q$ - axes stator flux linkages and $i_{d s}^{r}$ and $i_{\psi_{s}^{r}}^{r}$ are the $d$ - and $q$ - axes currents in a reference frame synchronous with the rotor, respectively. Assuming a similar behavior of $\lambda_{d s}^{r}$ and $\lambda_{q s}^{r}$ in the stator and rotor cores, as it will be shown later, radial and tangential leakage flux components, $\lambda_{l d s}^{r}$ and $\lambda_{l q s}^{r}$ are given by the interaction of the coil flux and permanent magnet flux and can be measured using Hall-effect sensors. The leakage flux measured by the Hall-effect sensors will be labeled as $B_{\text {Ham }}$, $B_{H b m}$ and $B_{H c m}$, where subindex $m$ stands for $x$ or $y$ component. From the three measurements a leakage flux complex vector $\vec{B}_{\text {ldqs }}^{s}(2)$ in the stator reference frame is defined considering (3). The leakage flux complex vector in the stator reference frame $B_{\text {ldqs }}^{s}$ can be transformed to a rotor synchronous reference frame $\vec{B}_{\text {ldqs }}^{r}$ (4).

a)

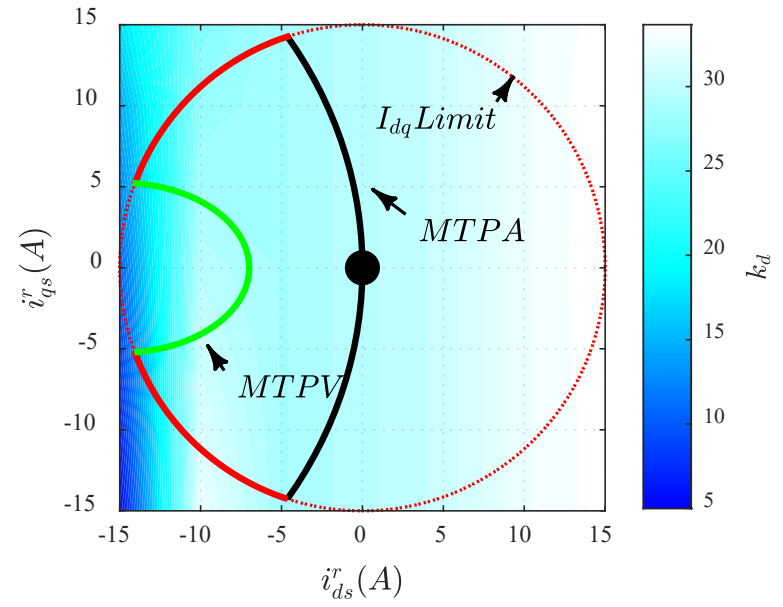

b)

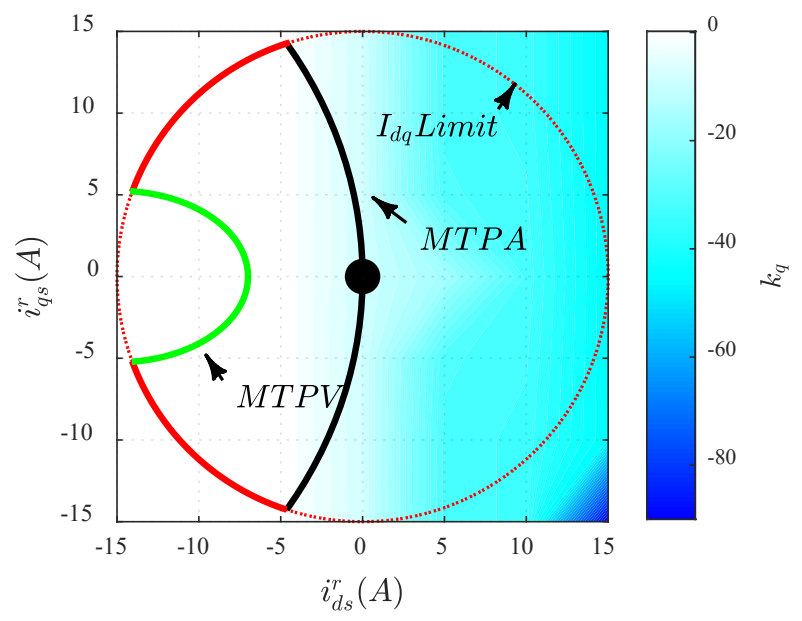

Fig. 4. FEA results of the relationship between stator flux linkage and leakage flux measured by the Hall-effect sensors for the IPMSM: a) $d$-axis $\left(k_{d}\right)$ and b) $q$-axis $\left(k_{q}\right)$.

The relationship between the stator flux linkage, $\vec{\lambda}_{\text {ldqs }}^{r}$, and the leakage flux density, $\vec{B}_{l d q s}^{r}$, measured by the Hall-effect sensors is given by the coefficients $k_{d}$ and $k_{q}$ as shown by (5) and (6) respectively; $k_{d}$ and $k_{q}$ as a function of $i_{d s}^{r}$ and $i_{q s}^{r}$ are shown in Fig. 4. It can be observed that $k_{d}$ is highly dependent on $d$-axis current but is barely affected by $q$-axis current (see Fig. 4a). $k_{q}$ in Fig. 4b) presents similar trends but is nearly constant in the second and third quadrants of the $d q$ plane. It is concluded from (5)-(6) and Fig. 4 that machine torque could be 
modeled as (7). $k_{d}$ and $k_{q}$ depend on the machine geometry, materials and sensor active area. In this work, they will be estimated experimentally from the measured leakage flux maps and torque.

For Operating conditions with low or no flux weakening operation, (i.e. negative $d$-axis current), the term $k_{d} B_{l d s}^{r} i_{q s}^{r}$ is dominant; $k_{d}$ can be considered as a constant under these working conditions, see Fig. 4a). For high flux weakening operation, the term $k_{q} B_{l q s}^{r} i_{d s}^{r}$ becomes dominant, $k_{q}$ can be assumed constant under these working conditions, see Fig. 4b).

$$
\begin{gathered}
T=\frac{3 p}{2}\left(\lambda_{d s}^{r} i_{q s}^{r}-\lambda_{q s}^{r} i_{d s}^{r}\right) \\
\vec{B}_{l d q s}^{s}=\frac{2}{3}\left(B_{H a m}+a B_{H b m}+a^{2} B_{H c m}\right) \\
a=e^{j 2 \pi / 3} \\
\vec{B}_{l d q s}^{r}=\vec{B}_{l d q s}^{s} e^{j \theta_{r}} \\
k_{d}=\frac{\lambda_{d s}^{r}}{B_{l d s}^{r}} \\
k_{q}=\frac{\lambda_{q s}^{r}}{B_{l q s}^{r}} \\
\hat{T}=\frac{3 p}{2}\left(k_{d} B_{l d s}^{r} i_{q s}^{r}-k_{q} B_{l q s}^{r} i_{d s}^{r}\right) \\
\varepsilon=\frac{T-\hat{T}_{T}^{T} \times 100}{T}
\end{gathered}
$$

The block diagram of the proposed torque estimation method is shown in Fig. 6. The inputs of the control block diagram are the measured leakage flux signals ( $B_{H a m}, B_{H b m}$ and $B_{H c m}$ ), which are low-pass filtered to remove high frequency induced noise. This filter is analog to prevent aliasing, with a cutoff frequency of $5 \mathrm{kHz}$. The flux leakage complex vector (2) is obtained afterwards and transformed to a rotor synchronous reference frame. The resulting $d$ - and $q$-axes leakage flux components are multiplied by the corresponding scaling factors and stator currents to provide the estimated torque (7).

\section{b. Implementation Issues}

Mounting errors can be due to axial displacement, radial displacement, or angular displacement [21].

Axial and radial displacement induces a variation of the magnetic flux density measured by the sensor both in $x$ - and $y$ axes directions, where the measured magnetic flux densities decrease as the sensor is moved further away from the magnet. This variation can be modeled as a variation of the gain of the sensor, which adds a positive and a negative sequence at $\pm \omega r$ to the fundamental component of the leakage flux complex vector, $\vec{B}_{\text {ldqs }}^{s}$. Angular displacement among sensors also adds a positive and a negative sequence at $\pm \omega r$ to the fundamental component of the leakage flux complex vector, $\vec{B}_{\text {ldqs }}^{s}$, but shifted $\pi / 2$ [21]. The positive and negative sequence will produce oscillations in both the d, $B_{l d s}^{s}$ and q, $B_{l d s}^{s}$ leakage flux components that will be reflected on the torque estimation. Fig. 5 shows the oscillations produced in the estimated torque when one sensor is shifted in the radial an axial direction up to $5 \mathrm{~mm}$ and when the sensor is shifted up to 15 electrical degrees.

a)

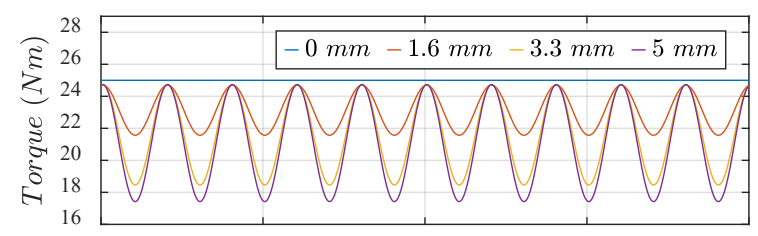

b)

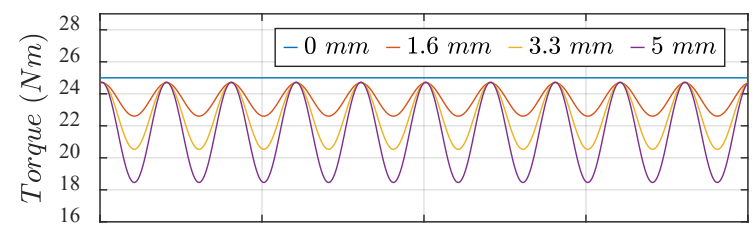

c)

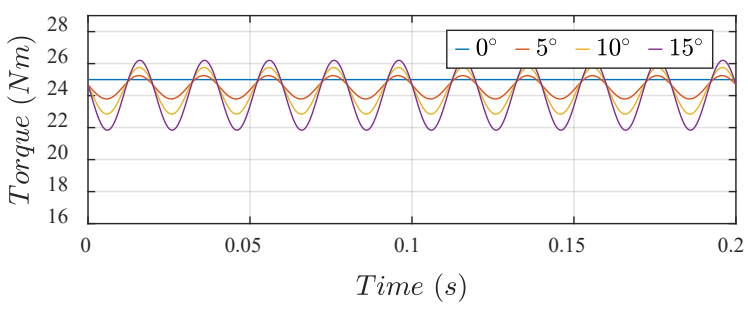

Fig. 5. Torque estimation for mounting errors in one sensor: a) radial displacement, b) axial displacement, and c) axial displacement (i.e., phase shift). $n=500 \mathrm{rpm}$.

Different magnetization state of the magnets due to assembling tolerances or partial (local) demagnetization may also affect the developed torque of the machine. When uniform demagnetization occur, the output torque of the machine will be reduced (for a given current). The proposed method will detect this variation, i.e. a uniform demagnetization does not affects to the method applicability. When non-uniform demagnetization occurs, the mean output torque of the machine will be slightly reduced (for a given current). The proposed method will detect this variation since the leakage flux will be reduced accordingly, i.e. a non-uniform demagnetization does not affects to the method applicability.

\section{EXPERIMENTAL RESULTS}

As discussed in Fig. 1, Hall-effect sensors [30] are attached to the end shield of the IPMSM and SPMSM as shown in Fig. 7a) and Fig. 7b) respectively. The test machines are fed by an inverter, the switching frequency is set to $10 \mathrm{kHz}$. A torque transducer (T25 from Interface) of $\pm 100 \mathrm{Nm}$ with a combined error (i.e. due to nonrepeatability and temperature) of $\pm 0.1 \%$ is used for torque measurement in both cases. The machines are mechanically coupled to a motion-controlled PMSM that will operate as a load. These machines will be experimentally evaluated using the test benches shown in Fig. 7c) and 7d), their main design parameters being shown in Table II. 


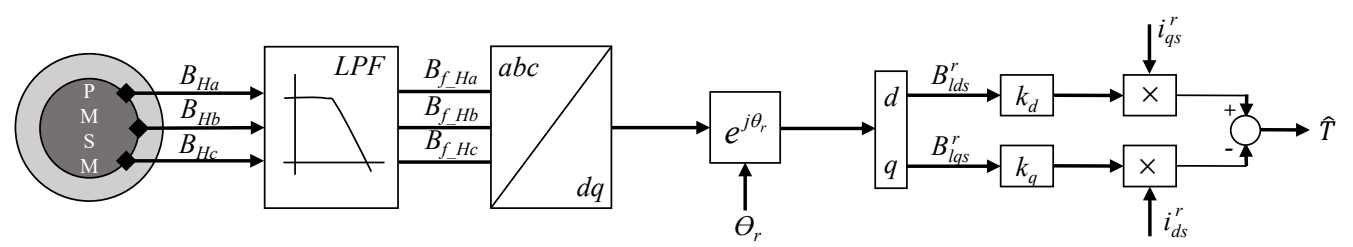

Fig. 6. Block diagram for torque estimation using leakage flux components.

a)

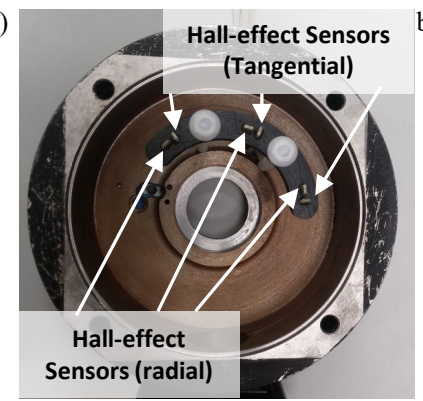

c)

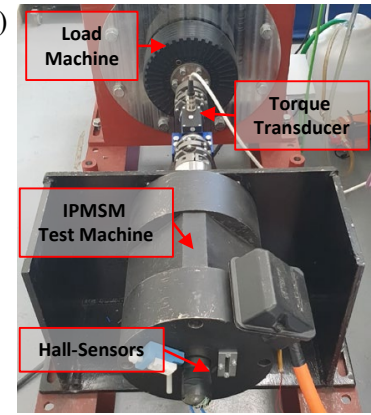

b)

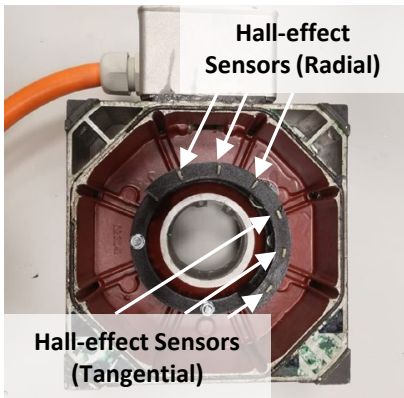

d)

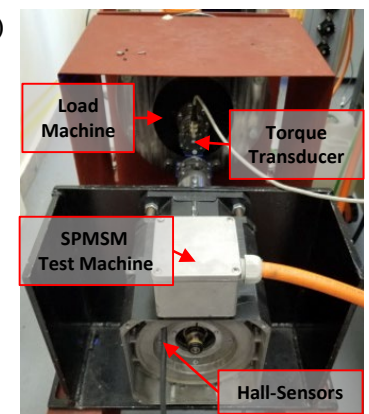

Fig. 7. Test machines: a) IPMSM end-shield with the hall-effect sensors, b) SPMSM end-shield with the hall-effect sensors, c) test bench for the IPMSM and d) test bench for the SPMSM.

Conventional linear Hall-effect sensors typically used in commercial PMSMs will be used in this work [30]. Although practical implementation of the method proposed can be achieved using only radial and tangential flux, an arrangement of two sets of three flux sensors capable of measuring both tangential and radial leakage flux has been installed in the motor end-shields as shown in Fig. 7a) and b). Experimental results will be conducted at a constant rotational speed, $n$, of $500 \mathrm{rpm}$; experimental results at different speeds are not included because the flux measurement is speed independent [26]. Magnet temperature variation has not been included in the study because it has been extensively studied in [26]. As shown in [26], as the PM temperature raise, both PM flux linkage and PM leakage flux decrease. The proposed method will estimate a lower torque as the PM temperature increases according to (7) and [26], so estimated torque will not be affected.

\section{a. Magnetic Flux density measurement}

Fig. 8 and 9 show the variation of $B_{l d s}^{r}$ and $B_{l q s}^{r}$ within the $I_{d q}$ plane for the IPMSM and the SPMSM shown in Fig. 1, respectively. As expected, $B_{l d s}^{r}$ decreases when flux weakening

current is applied (i.e. negative $i_{d s}^{r}$ ) while increases when flux intensifying current (i.e. positive $i_{d s}^{r}$ ) is applied. Fig. 8a) and Fig. 9a) also show a slight dependency of $B_{l d s}^{r}$ and $i_{q s}^{r}$, due to the cross coupling between the $d$ - and $q$ - axes. Fig. 8b) and Fig. $9 \mathrm{~b})$ show that $B_{l q s}^{r}$ is highly dependent on $i_{q s}^{r}$ in both machines, which was expected since $B_{l q s}^{r}$ is mainly due to $i_{q s}^{r}$. $B_{l q s}^{r}$ is slightly affected by $d$-axis flux due to cross coupling between axis as shown in Fig. 8b) and Fig. 9b).

a)

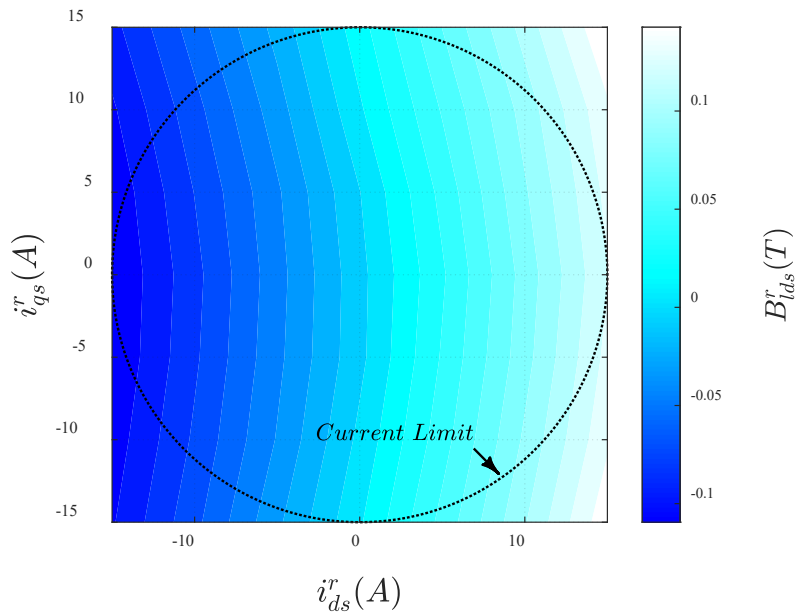

b)

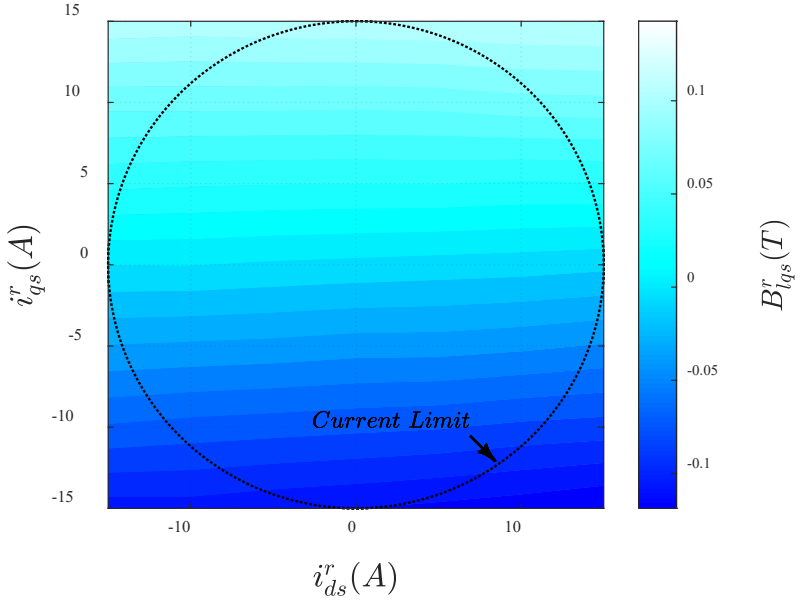

Fig. 8. - IPMSM experimental results: a) $d$-axis leakage flux measured in the $I_{d q}$ plane and b) $q$-axis leakage flux measured in the $I_{d q}$ plane. $n=500 \mathrm{rpm}$ and $I_{d q}$ step $=5 \mathrm{~A}$.

\section{b. Experimental results for the IPMSM}


The proposed torque estimation method is experimentally validated in the IPMSM shown in Fig. 1 and 7. For the experimental results shown in this section, the rotor speed is kept constant at $500 \mathrm{rpm}$ and $d$ - and $q$-axes currents are varied from $-1 \mathrm{pu}$ to $1 \mathrm{pu}$ in steps of $0.33 \mathrm{pu}$. Under these conditions, torque is measured using the torque transducer and estimated using the Hall-effect signals $\left(B_{H a}, B_{H b}\right.$ and $\left.B_{H c}\right)$. Color map in Fig. 10a) shows the measured torque on the $I_{d q}$ plane; a discontinuous line shows the limit for the current complex vector, the figure also shows machine's MTPA and MTPV trajectories.

a)

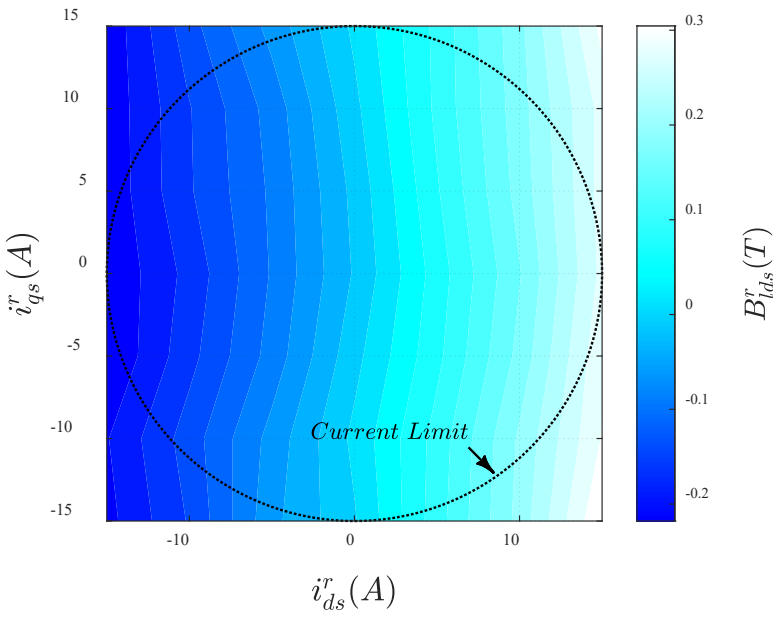

b)

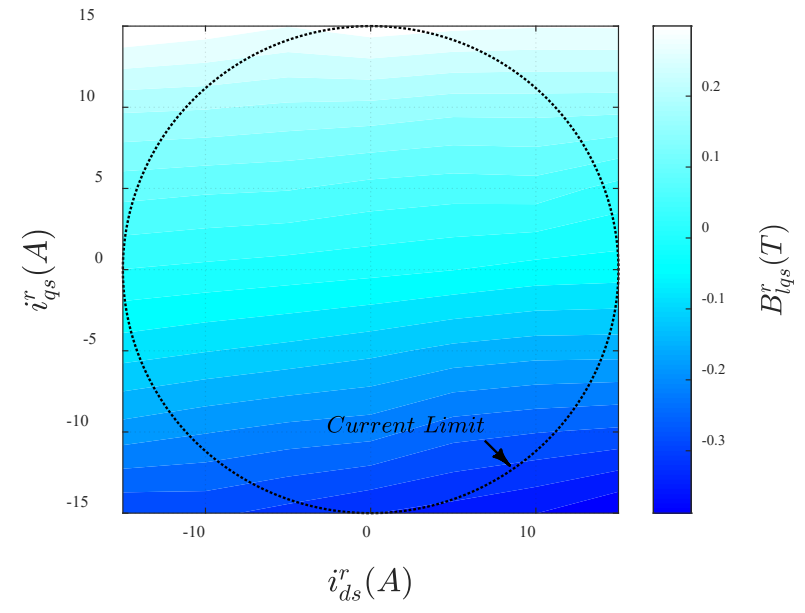

Fig. 9. - SPMSM experimental results. a) $d$-axis leakage flux measured in the $I_{d q}$ plane and b) $q$-axis leakage flux measured in the $I_{d q}$ plane. $n=500 \mathrm{rpm}$ and $I_{d q}$ step $=5 \mathrm{~A}$.

Fig. 10b) shows the estimated torque using (7) on the $I_{d q}$ plane. Fig. 11 shows the absolute torque error, i.e. difference between Fig. 9a) and 9b). A maximum error of $\pm 3 \mathrm{Nm}$ is observed, which is limited to -2.5 to $3.3 \mathrm{Nm}$ in the second and third quadrants of the $I_{d q}$ plane (i.e., machine can either operate as a motor or as a generator). In any case, estimation error is affected by both $i_{d s}^{r}$ It can be observed that the absolute error increases as the operating point moves along the MTPA curve and reaches its maximum at high flux weakening levels. If second or fourth quadrant are considered, the absolute estimation error $\pm 3 \mathrm{Nm}$. Fig. 11 also shows that the estimation error is affected by both $i_{d s}^{r}$ and $i_{q s}^{r}$ currents.

\section{c. Experimental results for the SPMSM}

The proposed torque estimation method is experimentally validated in an SPMSM equipped with the same Hall-effect sensors as the IPMSM and under the same working conditions. Fig. 12a) shows the resulting measured torque on the $I_{d q}$ plane using the torque transducer; a discontinuous line shows the limit for the current complex vector, the figure also shows machine's MTPA and MTPV trajectories. Fig. 12b) shows the resulting estimated torque on the $I_{d q}$ plane using (7). Fig. 13 shows the absolute error, the error varying from $\pm 3.2 \mathrm{Nm}$. Fig. 12 also shows that the maximum error was obtain when the machine is operating along the MTPA line (i.e. typical operating region of SPMSMs) is $\pm 0.5 \mathrm{Nm}$. The estimation error is barely affected by both $i_{d s}^{r}$ and $i_{q s}^{r}$ currents for the SPMSM.

a)

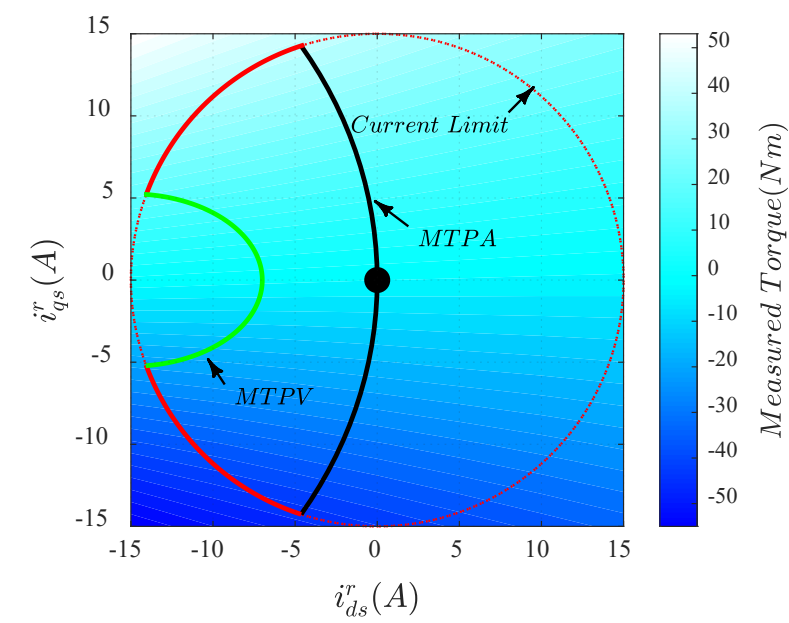

b)

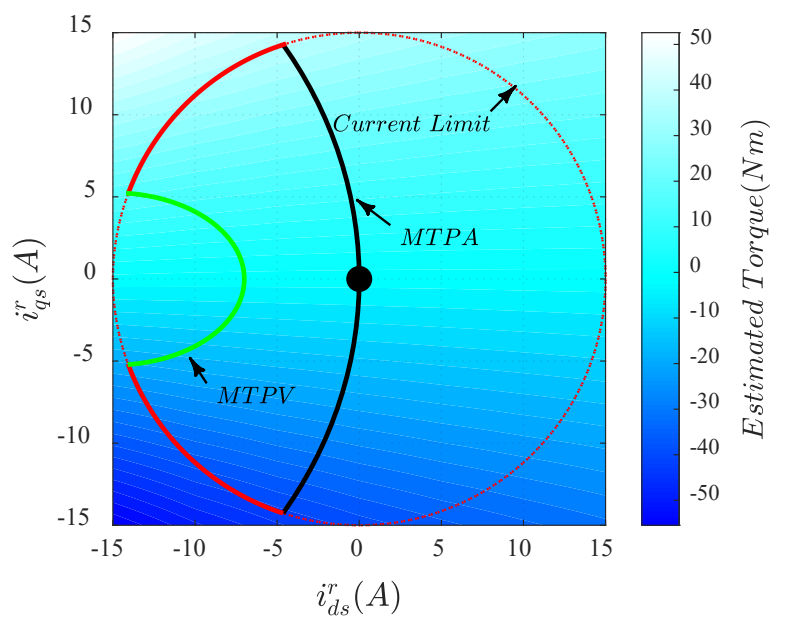

Fig. 10. IPMSM torque a) measurement using a torque transducer and b) estimated using the proposed method. Dotted lines delimit constant torque regions. $n=500 \mathrm{rpm}$ and $\mathrm{I}_{\mathrm{dq}}$ step $=5 \mathrm{~A}$. 


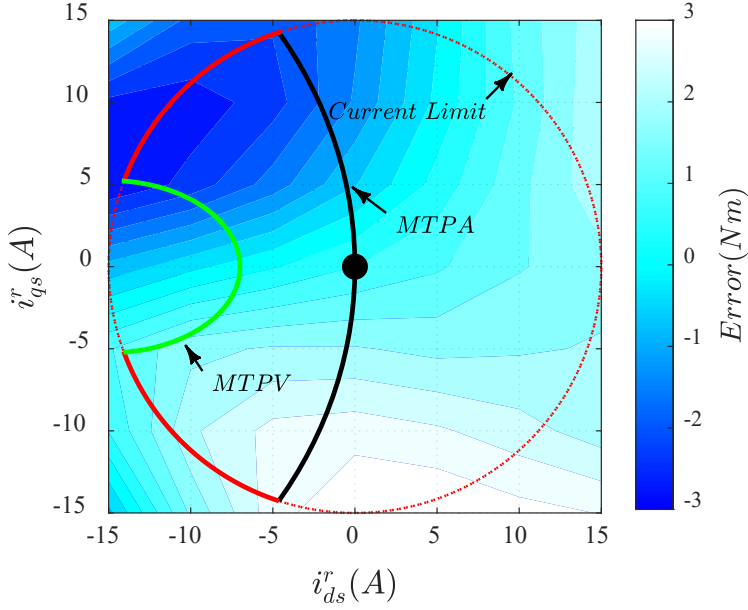

Fig. 11. IPMSM torque error. $n=500 \mathrm{rpm}$ and $I_{d q}$ step $=5 \mathrm{~A}$.

a)

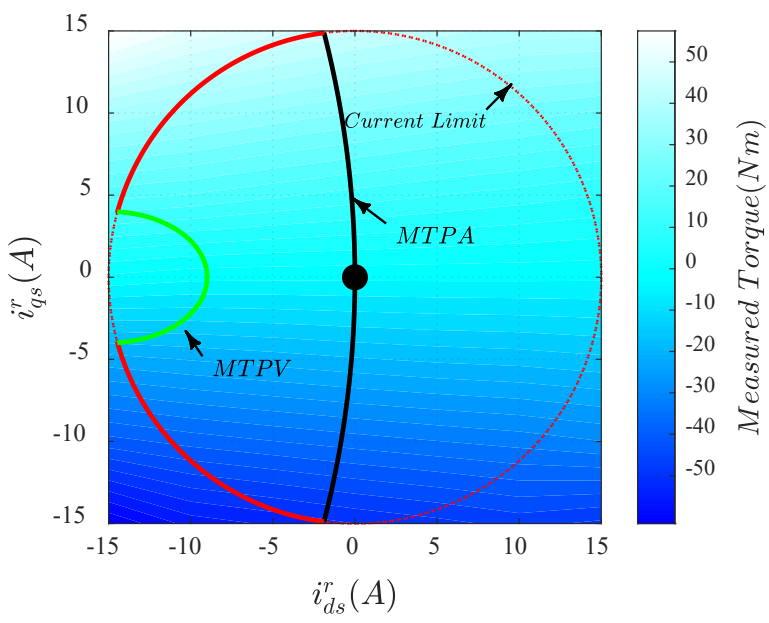

b)

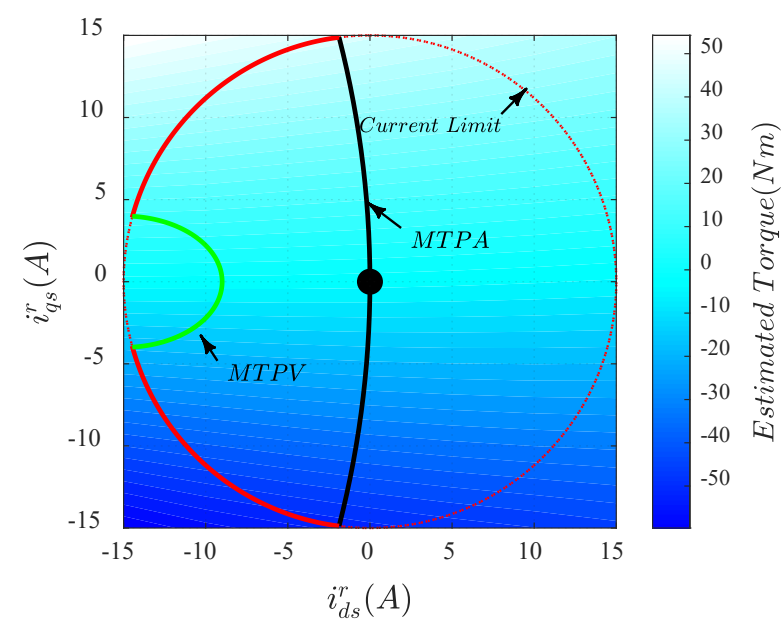

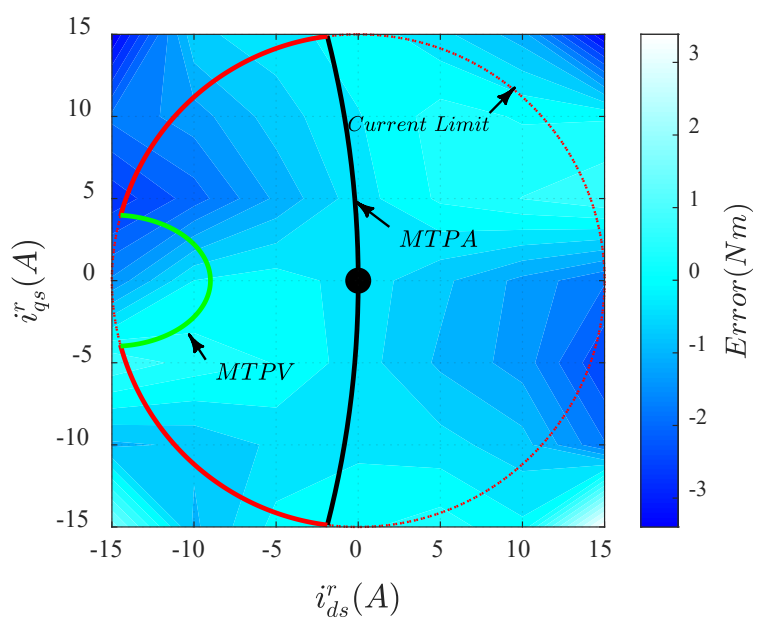

Fig. 13. SPMSM torque error. $n=500 \mathrm{rpm}$ and $i_{d q s}^{r}$ step $=5 \mathrm{~A}$.

a)

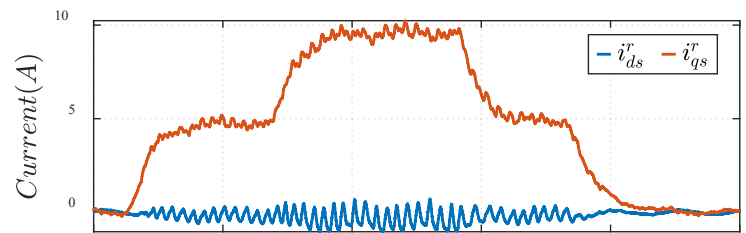

b)

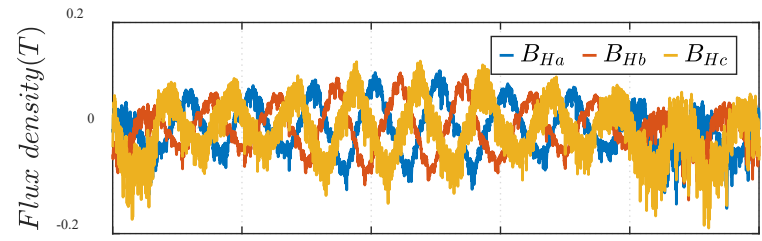

c)

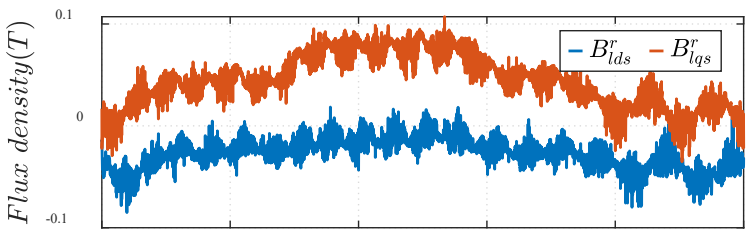

d)

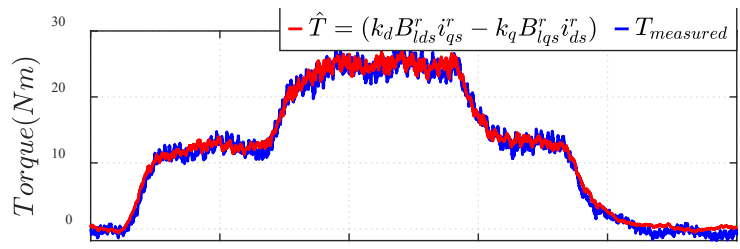

e)

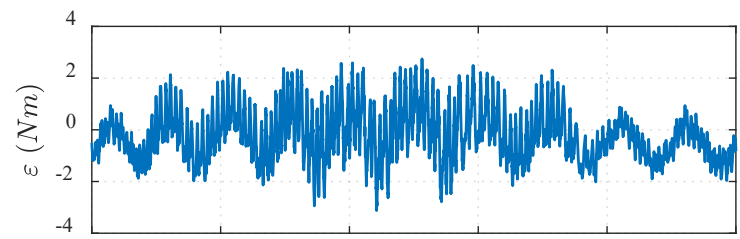

Fig. 12. SPMSM torque a) measurement using a torque transducer and b) estimated using the proposed method. Dotted lines delimit constant torque regions. $n=500 \mathrm{rpm}$ and $I_{d q}$ step $=5 \mathrm{~A}$ 


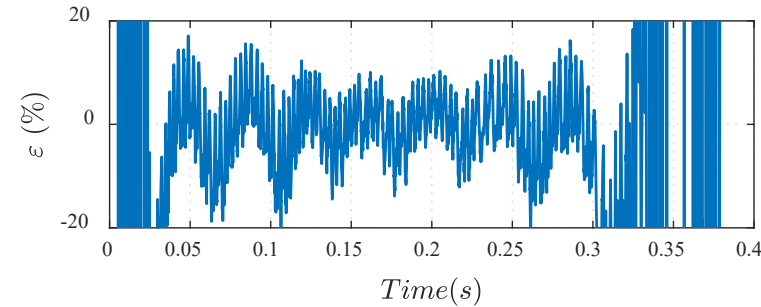

Fig. 14. Online torque estimation in an IPMSM for Iq current variation, a) current commands, b) measured flux signals, c) leakage flux complex vector components, d)measured and estimated torque e) estimation error (absolute), and d) estimation error (relative). $n=500 \mathrm{rpm}$.

\section{d. Online torque estimation}

The control block diagram shown in Fig. 6 is used for online torque estimation, which has been performed on the IPMSM shown in Fig. 1. Fig. 14 shows online toque estimation experimental results when Id current is set to zero and Iq varies with time. Fig. 14a) shows the $\mathrm{d}$ and $q$-axis currents, the measured leakage flux densities, $B_{H a}, B_{H b}$ and $B_{H c}$, are shown in Fig. 14b), while the leakage flux complex vector components are shown in Fig. 14c). The resulting measured and estimated torque based on (7) are shown in Fig. 14d), with $k_{q}=1.993$ and $k_{d}=31.323$. These values have been set to minimize the peak error when the machine is being operated in the second quadrant of the $I_{d q}$ plane, according to Fig. 4. The absolute estimation error is shown in Fig. 14e), which is seen to be within $\pm 2 \mathrm{Nm}$. Fig. $14 \mathrm{f}$ ) shows the relative error (8). As expected, the relative error tends to infinity when the actual torque is zero. It can also be observed that when the actual torque is different from zero, the estimated error remains within $\pm 10 \%$; according to Fig. 5, oscillations in Fig. 14 are due to assembling tolerances of the sensors in test bench.

Fig. 15 shows online toque estimation experimental results when Iq current is set to $10 \mathrm{~A}$ and Id varies with time. Fig. 15a) shows the $\mathrm{d}$ and q-axis currents, the measured leakage flux densities, $B_{H a}, B_{H b}$ and $B_{H c}$, are shown in Fig. $15 \mathrm{~b}$ ), while the leakage flux complex vector components are shown in Fig. 15c). The resulting measured and estimated torque based on (7) are shown in Fig. 15d), with $k_{q}=1.993$ and $k_{d}=31.323$ as in Fig. 14. The absolute estimation error is shown in Fig. 15e), which is seen to be within $\pm 3 \mathrm{Nm}$. Fig. 15f) shows the relative error (8). It can be observed that the estimated error remains in the $\pm 10 \%$ range. However, the average value is near zero, and according to Fig. 5, oscillations in Fig. 15 are due to assembling tolerances of the sensors in the machine.

a)

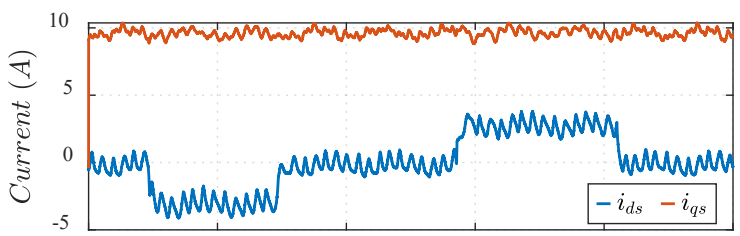

b)

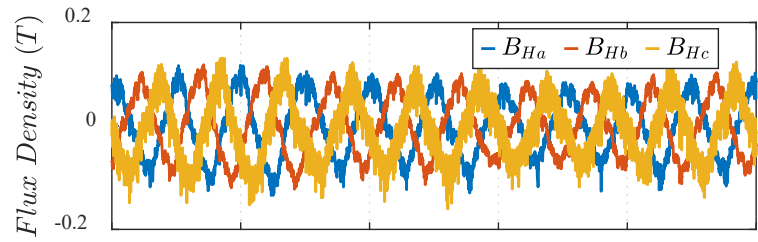

c)

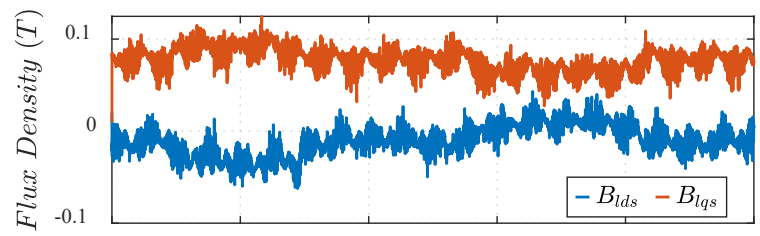

d)

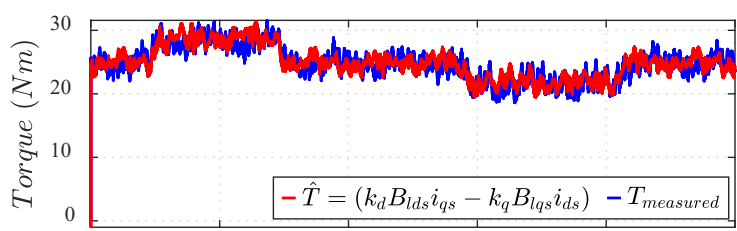

e)

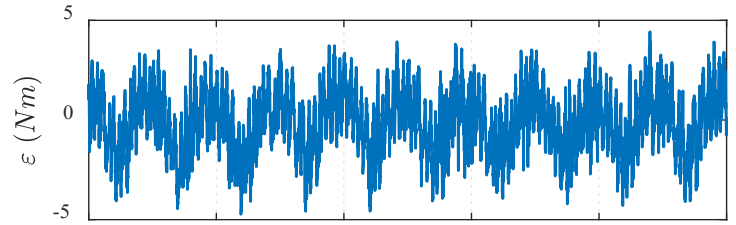

f)

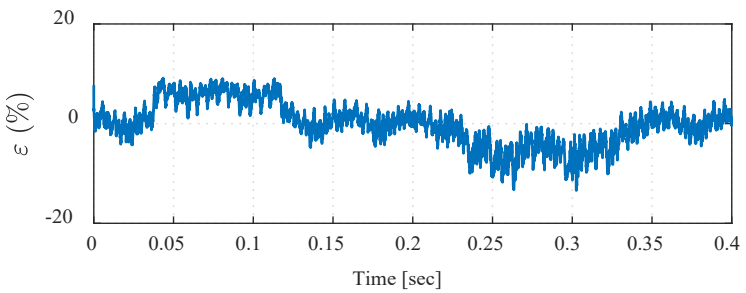

Fig. 15. Online torque estimation in an IPMSM for Id current variation, a) current commands, b) measured flux signals, c) leakage flux complex vector components, d)measured and estimated torque e) estimation error (absolute), and f) estimation error (relative). $n=500 \mathrm{rpm}$.

\section{CONCLUSIONS}

Torque estimation in PMSMs using Hall-effect sensors has been analyzed in this work. The proposed method relies on the measurements provided by linear Hall-effect sensors typically used in commercial PMSM drives for motion control. The flux measured by the Hall-effect sensors is used to define a leakage flux complex vector, which is used for torque estimation purposes. Experimental results have been presented for a SPMSM and an IPMSM to demonstrate the viability of the proposed method.

\section{ACKNOWLEDGMENT}

The authors would like to thank BOMATEC for the support providing magnet samples.

\section{REFERENCES}

[1] Lixin Tang, Limin Zhong, M. F. Rahman and Yuwen Hu, "A novel direct torque control for interior permanent-magnet synchronous machine drive 
with low ripple in torque and flux-a speed-sensorless approach," in IEEE Transactions on Industry Applications, vol. 39, no. 6, pp. 1748-1756, Nov.-Dec. 2003. doi: 10.1109/TIA.2003.818981

[2] Duane C. Hanselman, "Brushless Permanent Magnet Motor Design" New York: Mc-Graw Hill, 1994.

[3] C. Yu, T. Fukushige, N. Limsuwan, T. Kato, D. D. Reigosa and R. D. Lorenz, "Variable-Flux Machine Torque Estimation and Pulsating Torque Mitigation During Magnetization State Manipulation," in IEEE Transactions on Industry Applications, vol. 50, no. 5, pp. 3414-3422, Sept.-Oct. 2014, doi: 10.1109/TIA.2014.2305768

[4] F. Tinazzi and M. Zigliotto, "Torque Estimation in High-Efficency IPM Synchronous Motor Drives," in IEEE Transactions on Energy Conversion, vol. 30, no. 3, pp. 983-990, Sept. 2015, doi: 10.1109/TEC.2015.2408214.

[5] S. H. Kia, H. Henao and G. Capolino, "Torsional Vibration Assessment Using Induction Machine Electromagnetic Torque Estimation," in IEEE Transactions on Industrial Electronics, vol. 57, no. 1, pp. 209-219, Jan. 2010, doi: 10.1109/TIE.2009.2034181.

[6] N. Amano, "Method of calculating engine torque," Patent: US 20030204302 A1, 2004.

[7] Knauder, B., Savitski, D., Theunissen, J. et al. Electric torque vectoring for electric vehicles. ATZ Elektron Worldw 9, 50-55 (2014). https://doi.org/10.1365/s38314-014-0268-0

[8] M. Boisvert, P. Micheau, "Estimators of wheel slip for electric vehicles using torque and encoder measurements," Mechanical Systems and Signal Processing, vol. 76-77, 2016, pp 665-676, ISSN 0888-3270, https://doi.org/10.1016/j.ymssp.2016.02.017.

[9] H. Zhao, "Present Situation and Development Review of Torque Measurement", Applied Mechanics and Materials, Vol. 422, pp. 141-145, 2013

[10] Frank Umbach, Heinrich Acker, Johannes von Kluge, and Werner Langheinrich," Contactless measurement of torque," Mechatronics, vol.12, 2002,pp. 1023-1033

[11] P. Sue, D. Wilson, L. Farr and A. Kretschmar, "High precision torque measurement on a rotating load coupling for power generation operations," 2012 IEEE International Instrumentation and Measurement Technology Conference Proceedings, Graz, 2012, pp. 518-523. doi: 10.1109/I2MTC.2012.6229149

[12] J. Delvaux and P. Sue. "High Resolution Torque Measurement on a Rotating Shaft". US patent number 6795779, 2004.

[13] A. Niedworok, "Dynamic torque measurement of drive transmission in vehicles being in motion using strain gauges measurement technique and wireless data transmission," 2014 16th European Conference on Power Electronics and Applications, Lappeenranta, 2014, pp. 1-8. doi: 10.1109/EPE.2014.6910707

[14] K. C. Yeo, G. Heins and F. De Boer, "Comparison of torque estimators for PMSM," 2008 Australasian Universities Power Engineering Conference, Sydney, NSW, 2008, pp. 1-6.

[15] B. Cheng and T. R. Tesch, "Torque Feedforward Control Technique for Permanent-Magnet Synchronous Motors," in IEEE Transactions on Industrial Electronics, vol. 57, no. 3, pp. 969-974, March 2010.doi: 10.1109/TIE.2009.2038951

[16] Tong Liu, I. Husain and M. Elbuluk, "Torque ripple minimization with on-line parameter estimation using neural networks in permanent magnet synchronous motors," Conference Record of 1998 IEEE Industry Applications Conference. Thirty-Third IAS Annual Meeting (Cat. No.98CH36242), St. Louis, MO, USA, 1998, pp. 35-40 vol.1. doi: 10.1109/IAS.1998.732256
[17] X. Zhang and Z. Li, "Sliding-mode observer-based mechanical parameter estimation for permanent magnet synchronous motor," IEEE Transactions on Power Electronics, vol. 31, no. 8, pp. 5732-5745, 2016.

[18] Y. Feng, X. Yu, and F. Han, "High-order terminal sliding-mode observer for parameter estimation of a permanent-magnet synchronous motor," IEEE Transactions on industrial electronics, vol. 60 , no. 10, pp. 4272 4280,2013

[19] W. Xu and R. D. Lorenz, "High-frequency injection-based stator flux linkage and torque estimation for db-dtfc implementation on ipmsms considering cross-saturation effects," IEEE Transactions on Industry Applications, vol. 50, pp. 3805-3815, Nov 2014.

[20] M. Martinez, D. Reigosa, D. Fernandez, J.M. Guerrero and F. Briz, "PMSMs Torque Estimation Using Pulsating HF Current Injection", IEEE-SLED, pp. 96-101, Sept. 2018.

[21] D. Reigosa, D. Fernandez, C. González, S. B. Lee and F. Briz, "Permanent Magnet Synchronous Machine Drive Control Using Analog Hall-Effect Sensors," in IEEE Transactions on Industry Applications, vol. 54, no. 3 , pp. 2358-2369, May-June 2018. doi: 10.1109/TIA.2018.2802950

[22] X. Song, J. Fang and B. Han, "High-Precision Rotor Position Detection for High-Speed Surface PMSM Drive Based on Linear Hall-Effect Sensors," in IEEE Transactions on Power Electronics, vol. 31, no. 7, pp. 4720-4731, July 2016. doi: 10.1109/TPEL.2015.2479642

[23] Q. Ni et al., "A New Position and Speed Estimation Scheme for Position Control of PMSM Drives Using Low-Resolution Position Sensors," in IEEE Transactions on Industry Applications, vol. 55, no. 4, pp. 37473758, July-Aug. 2019, doi: 10.1109/TIA.2019.2904934.

[24] G. Liu, B. Chen and X. Song, "High-Precision Speed and Position Estimation Based on Hall Vector Frequency Tracking for PMSM With Bipolar Hall-Effect Sensors," in IEEE Sensors Journal, vol. 19, no. 6, pp. 2347-2355, 15 March15, 2019, doi: 10.1109/JSEN.2018.2885020.

[25] Z. Yu, M. Qin, X. Chen, L. Meng, Q. Huang and C. Fu, "Computationally Efficient Coordinate Transformation for Field-Oriented Control Using Phase Shift of Linear Hall-Effect Sensor Signals," in IEEE Transactions on Industrial Electronics, vol. 67, no. 5, pp. 3442-3451, May 2020, doi: 10.1109/TIE.2019.2922920.

[26] D. Fernandez et al., "Permanent Magnet Temperature Estimation in PM Synchronous Motors Using Low-Cost Hall Effect Sensors," in IEEE Transactions on Industry Applications, vol. 53, no. 5, pp. 4515-4525, Sept.-Oct. 2017. doi: 10.1109/TIA.2017.2705580

[27] Y. Park et al., "Online Detection and Classification of Rotor and Load Defects in PMSMs Based on Hall Sensor Measurements," in IEEE Transactions on Industry Applications, vol. 55, no. 4, pp. 3803-3812, July-Aug. 2019. doi: 10.1109/TIA.2019.2911252

[28] L. Dong, Y. Huang, J. Jatskevich and J. Liu, "Improved Fault-Tolerant Control for Brushless Permanent Magnet Motor Drives With Defective Hall Sensors," in IEEE Transactions on Energy Conversion, vol. 31, no. 2, pp. 789-799, June 2016, doi: 10.1109/TEC.2016.2526621.

[29] D. F. Alonso, Y. Kang, D. F. Laborda, M. M. Gómez, D. D. Reigosa and F. Briz, "Permanent Magnet Synchronous Machine Torque Estimation Using Low Cost Hall-Effect Sensors," 2019 IEEE 10th International Symposium on Sensorless Control for Electrical Drives (SLED), Turin, Italy, 2019, pp. 1-6, doi: 10.1109/SLED.2019.8896292.

[30] Allegro Microsystems, "Linear Hall-Effect Sensor ICs with Analog Output Available in a Miniature, Low-Profile Surface-Mount Package," A1308-0-DS, Rev.11 datasheet, Feb. 2020. 